\title{
Transnationalismusforschung: Höchste Zeit für mehr als Worte und Koeffizienten
}

\author{
Kommentar zum Beitrag „Warum pendeln Migranten häufig zwischen \\ Herkunfts- und Ankunftsregion" von Ludger Pries in Heft 1/2010 der Sozialen \\ Welt
}

\section{Von Frank Kalter}

Es wird viel publiziert dieser Tage und die Migrationsforschung ist sicherlich eines der Felder, die in diesem Sinne gerade eine besonders gute Konjunktur haben. Völlig zu Recht, muss man sagen, und die wesentlichen Gründe sind leicht zu erkennen: Zum einen fallen viele Probleme mit einer offensichtlichen und immer dringlicheren gesellschaftlichen Relevanz in diesen Themenbereich, zum anderen sind viele der sich um die Migration rankenden Phänomene auch aus rein sozialwissenschaftlicher Sicht faszinierend und stellen lohnende Herausforderungen an den generellen theoretischen und methodischen Wissensstand dar. Das Phänomen des sogenannten Transnationalismus, um das sich der Beitrag von Ludger Pries in Heft 1/2010 der Sozialen Welt dreht, gehört ganz ohne Zweifel dazu.

Es ist jedoch an der Zeit, dass wir in der Migrationsforschung über die ständige Selbstvergewisserung dieser Tatsachen hinauskommen und nach allen methodologischen Regeln der Kunst auch wirklich für einen kumulativen Erkenntnisfortschritt im Hinblick auf die zentralen Probleme bzw. Phänomene sorgen. Hiervon ist der genannte Beitrag weit entfernt. Vielmehr scheint er mir typisch für eine durchaus verbreitete Haltung in diesem Feld, dass es nämlich eben aufgrund der doch offensichtlichen Legitimation einer Beschäftigung mit bestimmten Fragen - schon mit reinen Begrifflichkeiten und vagen, eher assoziativen Andeutungen auf der theoretischen Ebene getan ist. Auf der empirischen Ebene werden dann eher illustrative und sehr willkürliche empirische Befunde hinzugefügt, weil sie, mit dem gleichen Ausgangsverständnis, für den Gegenstand einfach ,interessant " erscheinen. Der bereits vorhandene allgemeine theoretische Kenntnisstand wird dabei im Wesentlichen ignoriert, die methodischen Standards fallen nicht selten weit hinter die in anderen sozialwissenschaftlichen Anwendungsfeldern üblichen zurück.

Ich möchte versuchen, mein Unbehagen mit dem Beitrag von Ludger Pries näher zu erläutern, indem ich in aller Kürze die drei zentralen Kernpunkte einer jeden theoriegeleiteten empirischen Sozialforschung diskutiere: die Zielsetzung bzw. Fragestellung, den theoretischen Hintergrund und das konkrete empirische Vorgehen.

Im Hinblick auf die oben angedeutete Notwendigkeit eines Erkenntnisfortschrittes ist zunächst sehr zu begrüßen, dass in der Einleitung ausdrücklich betont wird, es gehe in dem Beitrag nicht - wie im überwiegenden Teil der bisherigen Transnationalismus-Literatur - um einen neuerlichen Existenzbeweis von Phänomenen, die man als ,transnational bezeichnen kann. Denn wer würde das bestreiten, und wer würde wirklich ernsthaft die vielzitierte, Container'-Vorstellung (Pries 2010: 85) vertreten? Der Aufsatz formuliert schon im Titel eine von ihrer Struktur her potentiell lohnende und klare Fragestellung: „Warum pendeln manche Migranten häufig zwischen Herkunfts- und Ankunftsregion?" Weniger klar bleibt, wo genau das Problem bzw. Rätsel ist, das sich hinter dieser Fragestellung verbirgt. Hier sind mindestens zwei sinnvolle Zusatzassoziationen denkbar, und beide werden im Verlauf des Textes, allerdings selten klar voneinander trennbar, verfolgt. Frage 1: Warum pendeln manche Migranten häufig zwischen Herkunfts- und Ankunftsregion und manche nicht? Das ist eine legitime, wenngleich noch nicht sonderlich fesselnde Problemstellung. Sehr viel reizvoller für die wei- 
tere Entwicklung im Forschungsfeld ist Frage 2: Warum pendeln manche Migranten häufig zwischen Herkunfts- und Ankunftsregion, obwohl man das (vor dem Hintergrund des bisherigen theoretischen Kenntnisstandes) so nicht erwarten würde? Zu keiner dieser Fragen kann der Aufsatz aber irgendeinen nennenswerten theoretischen oder empirischen Beitrag leisten. Schlimmer noch: Mit dem alleinigen Bezug auf die äußerst vage ,innovative“ (S. 69) transnationale Forschungsperspektive werden so gut wie alle Erkenntnisse verwässert bzw. ignoriert, die die bisherige Migrationsforschung als Antworten bereithält.

Zum theoretischen Aspekt: Die Einleitung kündigt an, „dass solche Migrationsphänomene am besten durch das Konzept der transnationalen Migration bzw. der Transmigration erklärt werden können“ (S. 70). Da wird man hellhörig, denn das wäre wohl das erste ,Konzept", das schon irgendetwas erklären könnte. Nach einem expliziten Theorieteil, der dies genauer erläutern und entsprechende erklärende Mechanismen darlegen würde, sucht man dann in der sehr ungewöhnlichen Gesamtstruktur des Beitrages aber vergebens. Alles was der Aufsatz in dieser Hinsicht bietet, ist folgende Passage, die die Titelfrage etwas modifiziert noch einmal expliziter aufgreift: ,Warum nehmen so viele Befragte das hohe Risiko und die enormen Kosten von wiederholten Landeswechseln zwischen den USA und Mexiko auf sich? (...) Antwort: Diese Menschen richten als Transmigranten ihre Erwerbsstrategien und häufig auch ihre familiäre Lebensgewohnheiten pluri-lokal zwischen Mexiko und den USA ein“"(S. 76). Ach so! Das ist nichts anderes als eine Umschreibung des Explanandums und damit ein Paradebeispiel für eine Scheinerklärung. Und dabei handelt es sich nicht etwa um ein nur unfair herausgegriffenes Text-Fragment, denn es geht im gleichen Stil weiter: „Die biographischen Lebensentwürfe und kollektiven Normalitätsstandards, das alltägliche Leben sowie der Lebensverlauf als eine Sequenz unterschiedlicher Beschäftigungen und Wohnsitze spannen sich in einem mehr oder weniger einheitlichen und dauerhaften Sozialraum über mehrere geographische Plätze hinweg auf" (S. 76). Das ist eine weitere, mit noch unklareren Begriffen versehene Umformulierung des Phänomens; das besitzt noch immer keinen Informationsgehalt. Und schließlich: „Durch dieses pluri-lokale und transnationale Leben können sie ihre Handlungschancen erweitern, und in der Regel empfinden sie diese transnationalen Lebensweisen nicht nur als Herausforderung, sondern auch als Bereicherung" (S. 76 f). Nun gut, das könnte man zumindest als Aufforderung verstehen, den Kern einer Erklärung grundsätzlich in einem weiten utilitaristischen Ansatz, und damit einem doch sehr , traditionellen' Paradigma zu suchen, aber das ist noch immer informationsleer. Und dies ist dann schon alles, was sich in diesem Beitrag explizit der ,Erklärung‘ widmet. Daraus ergibt sich weder irgendeine brauchbare Hypothese im Hinblick auf die Selektivität des Phänomens (Frage 1), noch ein Hypothese, die im Widerspruch zu bewährten erklärenden Ansätzen stände (Frage 2). Ärgerlich ist das nicht nur deshalb, weil leere Aussagen Zeit- und Papierverschwendung sind, sondern auch, weil die in allgemeineren Zusammenhängen der Migrationsforschung bewährten Erklärungsansätze eine Fülle von äußerst informationshaltigen Hypothesen über Frage 1 erlauben würden (und sich Frage 2 damit bis zum Beweis des Gegenteils erledigt hätte).

Da also jegliches informationshaltige Argument fehlt, ist es nicht verwunderlich, dass völlig unklar bleibt, was in dem zitierten Beitrag eigentlich das Ziel des empirischen Vorgehens ist. Die entsprechenden Teile bieten eine von ihrer Struktur her schwer durchschaubare Aneinanderreihung von Ergebnissen des zugrundeliegenden Forschungsprojekts, aus denen sich der Leser selbst eine Botschaft konstruieren muss. Bei vielen Befunden des dritten Teils (S. 74-77) bleibt dabei offen, auf welchen genauen Analyseverfahren oder gar Studienteilen (ereignisorientierter Datensatz oder autobiographische Interviews) sie beruhen. Das Leben der Familie Doña Rosa (S. 77-79) ist ja bunt und interessant, aber eingangs des Beitrags wurde doch betont, dass es eigentlich nicht wieder um neuerliche Existenzbeweise und reine Illustrationen transnationaler Phänomene gehen soll. Die multivariaten Analysen im vierten Teil (S. 79-84) sind Variablensoziologie in selten so entlarvender Reinform. Die Modelle sind vollkommen will- 
kürlich. Wie oben schon angedeutet wurde, ist keine einzige Variable aus einem übergreifenden zentralen theoretischen Argument abgeleitet. Um es an einem Beispiel noch einmal ganz deutlich zu machen: Leitend für Projekt und Artikel ist doch die sogenannte,, Hypothese sich herausbildender transnationaler Sozialräume“ (S. 71). Das bedeutet laut Autor: „Häufige (und nicht nur saisonale) Landeswechsel in der Erwerbskarriere sind demnach nicht unbedingt Ausdruck von gescheiterten Auswanderungs- bzw. Rückkehrwanderungsstrategien, sondern von transnationalen Arbeits- und Lebensstrategien. "Wenn man das tatsächlich als eine ,Hypothese" akzeptieren wollte, dann müsste zunächst irgendwo genau erläutert sein, was denn die Differentia specifica zwischen diesen beiden Strategien bildet. Und dann stellte sich die Frage: Welcher Koeffizient oder welche Koeffizienten in den multivariaten Analysen dienen hier als kritischer Test? Diese und andere zentralen Anschlussfragen werden aber nirgends gestellt, aufgegriffen oder gar beantwortet.

Die Analysen sind aber auch in rein deskriptiver Hinsicht (z.B. für Frage 1) wertlos. Die langatmigen empiristischen Interpretationen der Effektrichtungen und-stärken sind unbrauchbar, da die Modelle vollkommen fehlspezifiziert und die gewählten Methoden wenig adäquat sind. Warum zur Analyse wiederholter Ereignisse bei Vorliegen von Verlaufsdaten beispielsweise keine Methoden der Ereignisdatenanalyse verwendet werden, ist schwer nachvollziehbar - das wäre für diese Fragen wesentlich zielführender und ist auch in der Migrationsforschung mittlerweile Standard. Die mangelnde ,Repräsentativität" der Daten im Hinblick auf die angestrebte Grundgesamtheit problematisiert der Autor schon selbst. Auch hier ist bezeichnend, dass diese Probleme lediglich mit Worthülsen kaschiert werden: So „wurde mit der durchgeführten Untersuchung keine Repräsentativität angestrebt, wohl aber eine methodisch kontrollierte und bestimmte Bedingungsfaktoren kontrollierende Auswahl der Interviewpartner als Erhebungseinheiten“ (S. 74) - was immer das genau heißen soll, und welche statistischen Konsequenzen damit auch immer verbunden sein mögen. Schließlich ist auch die Definition der Grundgesamtheit, das heißt das grundsätzliche Design der Erhebung, mangelhaft: Wir finden hier das in der Migrationsforschung leider verbreitete Problem des Fehlens adäquater Referenzgruppen. Um die oben skizzierten Fragestellungen einigermaßen angemessen beantworten zu können, benötigte man sowohl erfolgreiche ,Normalmigranten“ in den USA als auch Nicht-Migranten in Mexiko - beide Gruppen wurden den Ausführungen zufolge nicht gesampelt.

Kurz: Der Autor berichtet hier relativ willkürliche, wenig elaborierte Befunde aus einem empirischen Forschungsprojekt und versucht sie mit vagen begrifflichen Spielereien eher assoziativ zu einem Aufsatz zu verbinden. Das hat, wie eingangs angemerkt, mit problem- und theoriegeleiteter empirischer Sozialforschung nur wenig zu tun. Von einem Fachzeitschriftenaufsatz erwarte ich, dass am Ende irgendetwas zumindest ein wenig klarer bzw. weiter ist als am Anfang, und dass dabei auf dem theoretischen und methodischen Stand der Forschung aufgebaut wird. Beides kann ich im hier diskutierten Beitrag nicht erkennen. Ich kann insgesamt auch nicht erkennen, wie das programmatische Ziel, ,die bewährten klassischen Untersuchungsansätze (...) weiterzuentwickeln und auszudifferenzieren“ (S. 85) erreicht werden soll, solange man scheinbar glaubt, mit der bloßen Benennung und Umschreibung entsprechender Phänomene sei schon irgendein (Mehr-)Beitrag zu ihrer Erklärung geleistet.

Nachbemerkung: Die Redaktion der Sozialen Welt hatte mich darum gebeten, den Beitrag von Ludger Pries zu begutachten, und dieser Kommentar hier beruht im Kern auf den Ausführungen des Gutachtens. Ich hatte die Redaktion somit auf meine starken Bedenken aufmerksam gemacht. Diese Bedenken wurden auch dem Autor mitgeteilt - er hat daraufhin keine einzige (!) Überarbeitung vorgenommen. Die Herausgeber der Sozialen Welt haben sich trotzdem mehrheitlich dazu entschieden den Beitrag abzudrucken. Das halte ich, vorsichtig formuliert, für eine sehr unübliche Praxis und für einen fahrlässigen Umgang mit der Zeit von 
Gutachtern. Ich danke jedoch für die Gelegenheit und die wiederholte Ermunterung meine Bedenken zumindest ex-post noch einmal zu äußern. Ich habe lange gezögert, das zu tun. Mir wäre es auf jeden Fall wesentlich lieber gewesen, der Beitrag von Ludger Pries wäre in dieser Form nie erschienen und dieser Kommentar hätte sich schon im Vorfeld erledigt.

Prof. Dr. Frank Kalter Lehrstuhl für Allgemeine Soziologie

Universität Mannheim Seminargebäude A5 68131 Mannheim kalter@uni-mannheim.de 